\title{
Maestría en Ordenamiento y Gestión del Territorio: Líneas de Investigación
}

\author{
VILMA LORENA OCHOA LÓPEZ \\ Facultad de Ciencias Espaciales \\ Universidad Nacional Autónoma de Honduras
}

\section{RESUMEN}

La Maestría en Ordenamiento y Gestión del Territorio es un Programa Académico de la Universidad Nacional Autónoma de Honduras, que desarrolla el Observatorio Astronómico Centroamericano de Suyapa, ahora Facultad de Ciencias Espaciales en colaboración con el Departamento de Geografía de la Universidad de Alcalá, España. Este programa se crea en el año 2005 y actualmente está desarrollando su Segunda Promoción. El Plan de Estudios de la Maestría comprende seis campos: Geografía, Geodesia, Cartografía, Sistemas de Información Geográfica, Percepción Remota, y Ordenamiento Territorial, teniendo como requisito de graduación la presentación y defensa de un Proyecto de Investigación como Tesis. Las líneas de investigación que se están desarrollando son Regionalización, Análisis espacial, Cartografía de recursos naturales, riesgos, incendios, recursos turísticos y atlas, Dinámica de cobertura y usos del suelo, Ecología y Unidades de paisaje, Redes geodésicas, Localización de equipamientos, Uso de teledetección en la creación de datos, Desarrollo de aplicaciones de programación, y Aplicaciones de ordenamiento territorial.

Palabras claves: Maestría en Ordenamiento y Gestión del Territorio, Líneas de Investigación, Proyectos de Tesis.

\begin{abstract}
The Master in Land Management and Territory Ordering, is an Academic Program of the National Autonomous University of Honduras, developed at the Central American Suyapa Astronomical Observatory, now Space Science Faculty, in collaboration with the Department of Geography, University of Alcalá, Spain. This program was created in 2005 and is currently developing its second promotion. The curriculum of the Master consists of six fields: Geography, Geodesy, Cartography, GIS, Remote Sensing and Land Management, having as graduation requirement a Graduate Research Project as thesis. The research lines being developed are: Regionalization, spatial analysis, natural resource mapping, hazards, fires, tourism resources and atlases, cover dynamics and land use, ecology and landscape units, geodetic networks, location of equipment, remote sensing data use in the creation, application, development programming and land management applications.
\end{abstract}

Key words: Master in Land Management and Territory Ordering, Research Interests, Projects Thesis. 


\section{ORIGEN y APROBACIÓN DEL PROGRAMA DE MAESTRÍA}

La Maestría en Ordenamiento y Gestión del Territorio (MOGT) es un Programa de Maestría que nace en 2004, como una iniciativa del Observatorio Astronómico Centroamericano de Suyapa de la Universidad Nacional Autónoma de Honduras (OACS/UNAH) en colaboración con el Departamento de Geografía la Universidad de Alcalá (UAH), España y posteriormente se incorpora al proceso de creación la Secretaría de Gobernación y Justicia, a través de la Unidad Coordinadora de Proyectos (UCP).

Este Programa se creó en Septiembre de 2005, por Acuerdo de la Comisión de Transición y por Acuerdo del Consejo de Educación Superior - UNAH el cual aparece en el Libro de Registro de Aprobación de Carreras y Planes de Estudio de la Dirección de Educación Superior, en el Tomo IV- 05, Folio No.149, Registro RP-239-1005 .

La Maestría en Ordenamiento y Gestión del Territorio es un programa académico, cuya finalidad es formar profesionales calificados y comprometidos socialmente, para desempeñarse con eficacia y eficiencia en la dirección de los procesos de ordenamiento territorial integrado que se realizan en Honduras y en la Región Centroamericana. Además, es una respuesta a las necesidades de las diferentes instituciones gubernamentales, municipales y privadas de formar y capacitar recursos humanos en Tecnología Espacial y de la Comunicación para la gestión del territorio y manejo de los recursos naturales (UNAH, UAH, SGJ-UCP, 2005).

Previo a la preparación y aprobación del Plan de Estudios de la Maestría en Ordenamiento y Gestión del Territorio, se desarrolló un proceso de diagnóstico que señalo la necesidad de orientar el Plan hacia:

- La gestión de los recursos naturales, relacionada con áreas protegidas, producción y conservación de los recursos hídricos y bosques.

- La cartografía y gestión de riesgos, orientada a incendios, inundaciones, deslizamientos y otros fenómenos naturales.

- El ordenamiento territorial, orientado a la zonificación del territorio, el uso del suelo (actual y potencial), urbanización que se puede relacionar con la ubicación de infraestructura y servicios, y áreas de producción agrícola.

Habiendo sido éste ultimo el que resultó ser de interés prioritario, como consecuencia de la aprobación reciente, en ese entonces, de la Ley y el Reglamento de la Ley de Ordenamiento Territorial de Honduras.

\section{PLAN DE ESTUDIOS Y POLÍTICAS ACADÉMICAS}

En respuesta a las necesidades de país, establecidas en el Documento de Diagnóstico, se definió un Plan de Estudios para una Maestría Académica, que tiene como bases las Ciencias y Tecnologías Espaciales con aplicación al Ordenamiento Territorial. Este Plan de Estudios cuenta con 51 unidades valorativas (créditos) y 19 asignaturas, distribuidas en seis (6) áreas del conocimiento: (1) Geografía, (2) Geodesia, (3) Cartografía, (4) Sistemas de Información Geográfica, (5) Percepción Remota, (6) Ordenamiento Territorial.

Con las primeras cinco áreas, se espera que los estudiantes dominen conocimientos y técnicas espaciales fundamentales para el procesamiento, interpretación y análisis de datos geoespaciales y su aplicación en la ordenación del territorio. Y al desarrollar el área de Ordenamiento Territorial, los estudiantes adquirirán los 
conocimientos y habilidades necesarias para la administración, planificación y gestión territorial a nivel nacional, regional, municipal y local.

El Plan de Estudios tiene una carga horaria de 1788 horas de estudio, de las cuales el 81\% corresponde a las asignaturas ( $26 \%$ horas de teoría y $74 \%$ horas prácticas) y el $19 \%$ corresponde al proyecto de investigación (con $86 \%$ de horas prácticas).

Las asignaturas del Plan de Estudios de la Maestría siguen el flujograma que se muestra en la Figura 1. Aquí se muestra, por ciclos el orden de las 21 asignaturas que los estudiantes tienen que aprobar.

FIGURA 1. Flujograma de la Maestría en Ordenamiento y Gestión del Territorio de la UNAH.

Fuente: Plan de Estudios MOGT-OACS/UNAH)

\section{MOGT (5IU.V.)}

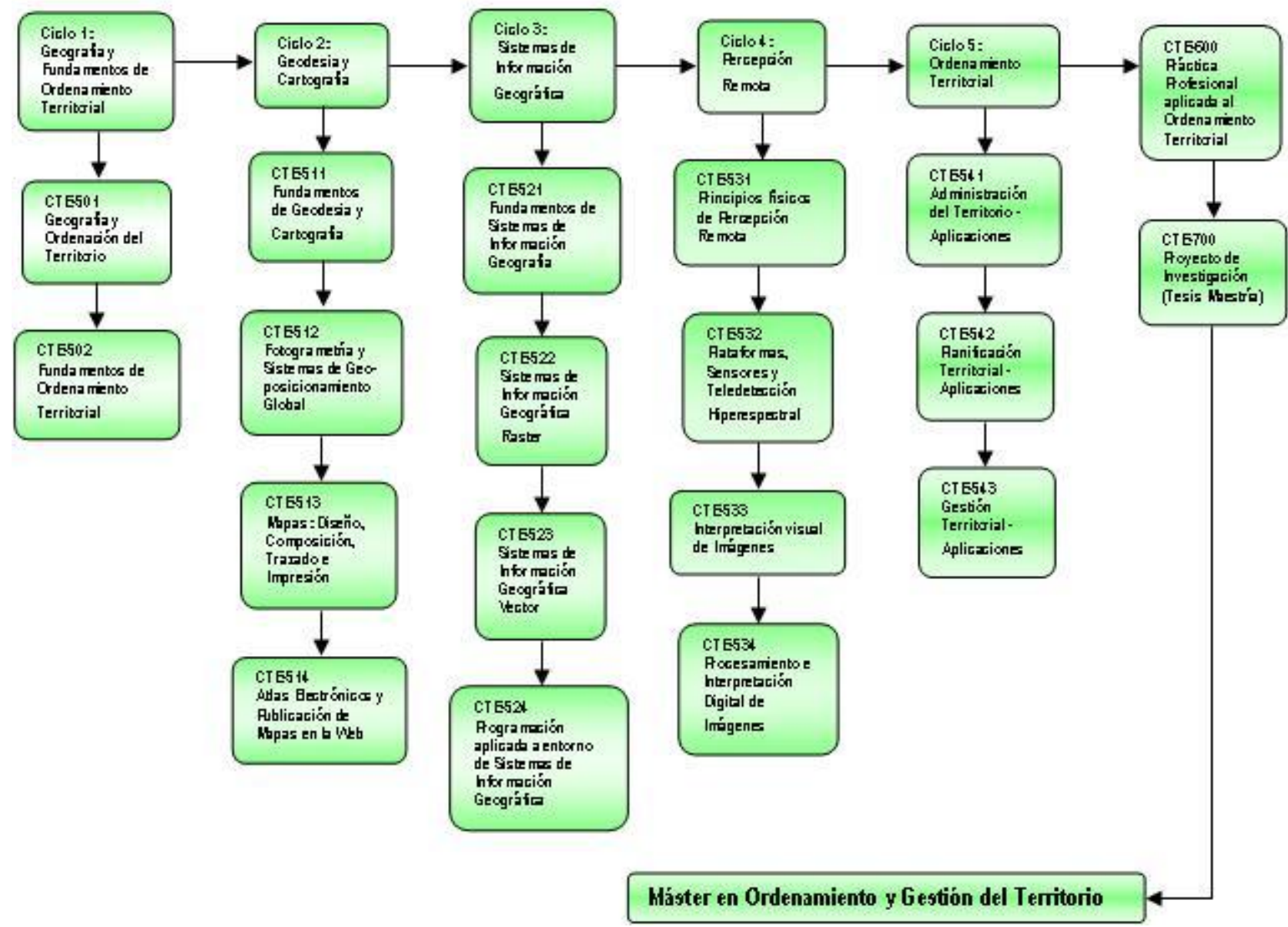

Para mantener la permanencia en el Programa de la MOGT, las políticas académicas exigen que los estudiantes cumplan con el $80 \%$ de asistencia, alcancen la nota mínima de aprobación de $80 \%$ y cumplan todos los requisitos académicos y financieros establecidos para los estudiantes universitarios en la UNAH. 


\section{POLÍTICAS DE INVESTIGACIÓN}

La Maestría en Ordenamiento y Gestión del Territorio tiene como Misión, "Formar profesionales responsable capaces de vincular los conocimientos teóricos-prácticos y la investigación de la ciencia y tecnología espacial con la realidad social y el medio natural, y que saben aplicarlos de manera autocrítica y propositiva a situaciones administrativas, de gestión y ordenamiento del territorio, para integrarse a los procesos de desarrollo de la nación". Su Visión es, "Dar respuesta a las necesidades nacionales y regionales, en los procesos de dirección, administración, gestión y ordenamiento del territorio". En base a lo anterior y a las líneas de investigación institucionales, dentro del Programa de Maestría se establecen las siguientes políticas de investigación:

- Fortalecer los objetivos de Docencia, Investigación y Extensión de la UNAH.

- Establecer Redes, entre la UNAH y otras universidades del mundo, para intercambiar experiencias y conocimientos científicos.

- Formar profesionales capaces de dirigir el proceso de Ordenamiento Territorial a nivel local, regional y nacional.

- Proponer alternativas de solución a los principales problemas que el Estado de Honduras enfrenta con la implementación de la Ley de Ordenamiento Territorial.

- Fortalecer la capacidad técnica de las instituciones responsables del Ordenamiento y Gestión del Territorio de Honduras.

- Contribuir al uso y aprovechamiento sostenible del recurso suelo y otros recursos naturales, además contribuir al rescate del patrimonio natural y arqueológico de la nación, a través de la ordenación del territorio.

\section{METODOLOGÍA}

El Plan de Estudios de la Maestría en Ordenamiento y Gestión del Territorio, se desarrolla a través de las asignaturas, los seminarios, la práctica profesional y el proyecto de tesis.

Las asignaturas. El plan de estudios se desarrolla en modalidad semipresencial, que consiste en que cada asignatura se desarrolla en un periodo de cinco semanas: cuatro semanas de clases en línea a través de la plataforma tecnológica educativa Moodle y una semana de clases presenciales en la ciudad universitaria. Durante las clases en línea los estudiantes revisan y analizan material bibliográfico, y participan en foros de discusión, todo esto bajo la coordinación de un profesor especialista en el área. Las clases presenciales están orientadas a reforzar la comprensión de los conocimientos teóricas de la asignatura y el desarrollo de sus aplicaciones prácticas.

Los seminarios. Simultáneamente a las clases presenciales, se desarrollan los Seminarios de Ordenamiento Territorial, que cuentan con la participan diferentes expositores, Profesores, estudiantes de postgrado e invitados especiales, que contribuyen al programa de formación. Con ellos se da a conocer a la comunidad en general los avances científicos relacionados con el Ordenamiento Territorial y campos afines. Además, se desarrollan los Seminarios de Investigación como un espacio de divulgación y discusión de las metodologías y técnicas de investigación científica; estos seminarios son desarrollados por todos y cada uno de los profesores del Programa. 
Práctica Profesional. Esta actividad de Vinculación Universidad - Sociedad, es un trabajo de aplicación desarrollado en base a casos reales de administración y gestión territorial a nivel nacional, regional, municipal y particularmente en zonas de interés geográfico y/o sectorial o temático, implicando el uso de SIG y Percepción Remota y sus tecnologías asociadas, que puede incluir trabajo de campo cuando sea necesario.

Proyecto de Tesis. Este se desarrolla bajo la dirección de un Profesor Tutor y con el apoyo de otros profesores asesores. El estudiante desarrolla un proyecto de investigación de su elección, donde aplica los conocimientos, las metodologías y técnicas de investigación para solucionar un problema específico de estudio, que puede ser de interés nacional, regional, municipal y particularmente en zonas de interés geográfico y/o sectorial o temático.

\section{PERSONAL DOCENTE}

En el Programa participan profesores especialistas, con amplia experiencia docente y en su campo profesional, además de su experiencia en la dirección o participación en proyectos de investigación científica y trabajo con estudiantes de postgrado.

La mayor parte de los profesores son profesionales contratados en calidad de Profesores Visitantes, de los cuales el $19 \%$ son nacionales y el $81 \%$ son extranjeros. Hasta ahora, estos últimos se desempeñan como profesores de las Universidades de Alcalá, Politécnica de Madrid y Del País Vasco de España, ó de la Universidad Nacional de Lujan, Argentina, la Universidad de Guadalajara de México y de otras universidades de la región, con las cuales existen nexos de colaboración. En la tabla 1, se presenta la lista de los profesores que participan en la MOGT destacando su área de especialización. Aquí se puede notar que los campos más representados son los Sistemas de Información Geográfica, Teledetección y sus aplicaciones. 
Tabla 1. Área de especialización del Personal Docente de la Maestría en Ordenamiento y Gestión del Territorio.

\begin{tabular}{|l|l|l|}
\hline No. & Profesores & Áreas de Especialización \\
\hline & Nacionales & \\
\hline $\mathbf{1}$ & $\begin{array}{l}\text { Dra. María Cristina Pineda de } \\
\text { Carías }\end{array}$ & Percepción Remota (Teledetección) \\
\hline $\mathbf{2}$ & Dra. Elsa Lily Caballero & Ordenamiento Territorial \\
\hline $\mathbf{3}$ & M.Sc. Vilma Lorena Ochoa López & Recursos Naturales \\
\hline $\mathbf{4}$ & M.Sc. Carlos Héctor Sabillón & Geografía \\
\hline $\mathbf{5}$ & Máster Jorge Reyes Silva & Administración \\
\hline & Internacionales & \\
\hline $\mathbf{6}$ & Dr. Joaquín Bosque Sendra & Geografía Humana \\
\hline $\mathbf{7}$ & Dr. Gustavo Buzai & Sistemas de Información Geográfica \\
\hline $\mathbf{8}$ & Dr. Francisco Maza Vázquez & Geodesia y Cartografía \\
\hline $\mathbf{9}$ & Dra. Ruth Miranda Guerrero & Atlas, Cartografía Temática y Multimedia, Gestión Territorial y \\
& & Tecnologías de la Información Geográica \\
\hline $\mathbf{1 0}$ & Dr. Aitor Bastarrica Izaguirre & Programación en Aplicaciones en SIG \\
\hline $\mathbf{1 1}$ & Dr. Juan Gregorio Rejas Ayuga & Teledetección y Análisis Hiperespectral, Modelos Digitales del Terreno \\
\hline $\mathbf{1 2}$ & Dr. José Antonio Malpica & Geodesia, Cartografía Matemática, Fotogrametría \\
\hline $\mathbf{1 3}$ & Dr. Francisco Escobar Martínez & Análisis Geográfico Regional \\
\hline $\mathbf{1 4}$ & Dr. Luis Carvacho Bart & Sistemas de Información Geográfica \\
\hline $\mathbf{1 5}$ & Dra. Guadalupe Rodríguez & Ingeniería Cartográfica, Geodesia y Fotogrametría \\
\hline $\mathbf{1 6}$ & Dr. Emilio Chuvieco Salinero & Análisis Geográfico Regional y Teledetección \\
\hline $\mathbf{1 7}$ & Dr. Víctor Rodríguez Espinosa & Geografía Humana \\
\hline $\mathbf{1 8}$ & Dra. Angnes Teresa Aldana & Geografía, Producción Cartográfica \\
\hline & Dezzeo & Teledetección \\
\hline $\mathbf{1 9}$ & Dra. Marta Yebra Alvarez & Teledetección \\
\hline $\mathbf{2 0}$ & Dra. Haydee Karszenbaum & Teledetección \\
\hline $\mathbf{2 1}$ & Dra. Patricia Oliva & Geografía Humanas, Geodesia y Topografía \\
\hline $\mathbf{2 2}$ & Dr. Wenseslao Plata Rocha & Desarrollo de Páginas Web e Infraestructura de datos Espaciales \\
\hline $\mathbf{2 3}$ & Dr. Diego José Padrón Paredes & Ordenamiento Territorial \\
\hline $\mathbf{2 4}$ & Arq. Osvaldo Ramacciotti & Rdministración de Tierras \\
\hline $\mathbf{2 5}$ & Arq. Edgardo Derbes & \\
\hline $\mathbf{2 6}$ & M.Sc. Carlo Foletti & \\
\hline & & Recursos Naturales \\
\hline
\end{tabular}

Además, participan otros profesores en calidad de invitados especiales, que desarrollan temas muy particulares en el marco de las asignaturas.

\section{LÍNEAS DE INVESTIGACIÓN}

Las áreas de investigación prioritarias que se plantean en el Plan de Estudios, y que se proponen desarrollar a través de los Proyectos de Investigación y Trabajos de Tesis del Programa de Maestría, surgen de la consulta 
con el Sector Gubernamental y otras instituciones relacionadas. Encontrándose que a nivel nacional las áreas de mayor relevancia son las siguientes:

- Regionalización nacional, esquema de ordenamiento territorial: A nivel nacional las diferentes instituciones han establecido las regiones más convenientes para el cumplimiento de sus objetivos, pero como nación no existe una estructura que estandarice las regiones.

- Áreas protegidas, zona núcleo y área de amortiguamiento: Las áreas protegidas que existen en el país, no cuentan en su totalidad con un decreto que las proteja, por lo que se hace necesario desarrollar investigaciones que generen los elementos adecuados para lograr un mejor manejo y administración de estas áreas.

- Cuencas, sub-cuencas y microcuencas: En regiones del país las cuencas, sub cuencas y microcuencas se están considerando como unidades de manejo del territorio y los recursos naturales, y algunas instituciones gubernamentales han establecido lineamientos para el aprovechamiento de estas áreas.

- Diagnóstico territorial municipal, zonificación: Su importancia radica en el apoyo que se pueda brindar a aquellos municipios de escasos recursos.

- Delimitación de perímetros urbanos: Desarrollar esta línea de investigación será una contribución importante para las comunidades.

- Zonificación urbana, centros históricos y sitios arqueológicos: La expansión urbana trae consigo la modernización de las comunidades y la pérdida de valiosos recursos históricos, esta línea de investigación puede crear elementos importantes para la identificación, valoración y preservación de estos recursos.

- La gestión de los recursos naturales, relacionada con áreas protegidas, producción y conservación de los recursos hídricos y bosques: Las investigaciones en este campo pueden contribuir a minimizar los impactos que el avance de las fronteras agrícola y urbana está causando en los recursos naturales.

- Cartografía y gestión de riesgos, orientada a incendios, inundaciones, deslizamientos y otros fenómenos naturales 0 antrópicos: La vulnerabilidad de Honduras a riesgos de incendios y fenómenos naturales plantea la necesidad de producir cartografía de alto nivel, que permita tomar decisiones más adecuadas para minimizar la exposición al riesgo de población y la infraestructura del país.

- Ordenamiento territorial, orientado a la zonificación del territorio, el uso del suelo (actual y potencial), urbanización que se puede relacionar con la ubicación de infraestructura y servicios, y áreas de producción agrícola.

Las investigaciones que se generen en el marco de la Maestría en Ordenamiento y Gestión del Territorio, pueden ser a partir de los Proyectos de Investigación de Tesis o como Proyectos de Investigación de los Profesores. En todos los casos, se espera que los resultados de la investigación generen teorías, información y herramientas que sirva de base para la toma de decisiones en las diferentes instituciones y organizaciones que participan en la administración y gestión del territorio y los recursos naturales de nuestro país y la región centroamericana.

\section{PROYECTOS DE TESIS}

Como requisito de graduación todo estudiante matriculado en el Programa de Maestría en Ordenamiento y Gestión del Territorio, debe desarrollar un proyecto de investigación, el cual finaliza con la elaboración y defensa de una tesis.

PRIMERA PROMOCIÓN MOGT1 
En el desarrollo del Seminario de Investigación se fueron realizando esfuerzos encaminados a que los estudiantes de la I Promoción de la MOGT definieran sus áreas de investigación. Pero fue durante la realización del I Congreso de Investigación Científica y como producto de las reuniones de trabajo de la Comisión Técnica de la Maestría con los estudiantes, que se formalizan los temas de Investigación. En la Tabla 2 se incluyen los temas de tesis con que se graduaron los estudiantes que lograron cumplir con todos los requisitos de graduación.

Tabla 2. Proyectos de Investigación de Tesis de los estudiantes: I Promoción MOGT.

\begin{tabular}{|c|l|}
\hline No. & Nombre del Proyecto de Investigación (Tesis) \\
\hline 1 & $\begin{array}{l}\text { Regionalización de Honduras: Hacia la construcción de Regiones-Plan a } \\
\text { través del análisis espacial cuantitativo }\end{array}$ \\
\hline 2 & $\begin{array}{l}\text { Análisis Multitemporal de la Cobertura de la Tierra en la Cuenca el Valle de } \\
\text { Jesús de Otoro, entre los años } 2000-2006, \text { aplicando técnicas de } \\
\text { teledetección. }\end{array}$ \\
\hline
\end{tabular}

Con las investigaciones propuestas se espera lograr los objetivos siguientes:

1. "Desarrollar tres propuestas de Regionalización Nacional, que sirvan de base para orientar el desarrollo social, económico y ambiental de Honduras, aplicando técnicas de análisis espacial cuantitativo".

2. "Generar geografía temática primaria utilizando datos multiespectrales en la cuenca del Valle de Jesús de Otoro, entre los años 2000 y 2006, con la finalidad de proporcionar información base que permita la toma de decisión en el ordenamiento y gestión del territorio".

\section{LÍNEAS DE INVESTIGACIÓN SEGUNDA PROMOCIÓN}

Para la Segunda Promoción de la Maestría en Ordenamiento y Gestión del Territorio (MOGT2), se han definido las aéreas de trabajo bajo las cuales los estudiantes desarrollaran su Proyecto de Investigación. Los Estudiantes de la MOGT2 con el apoyo de los Profesores del Comité Técnico de Investigación de la Maestría han identificado quince líneas de interés, y es de señalar la aparición de nuevas líneas a las establecidas en el Plan de Estudios:

- Localización de Equipamientos: El crecimiento poblacional, lleva implícito la ubicación adecuada de infraestructura que beneficie a más sectores de la población.

- Red Geodésica: El establecimiento de una red geodésica, es un recurso necesario para el desarrollo económico de cualquier región, ya que este es de vital importancia en la elaboración y actualización de cartografía de todo tipo y, muy en especial, en la gestión y ordenación del territorio.

- Dinámica de cobertura y uso del suelo: Estas áreas de investigación son importantes, pues son unos de los principales procesos generadores de cambios en los ecosistemas naturales. Honduras es un país donde la pérdida de la biodiversidad está asociada con la modificación de la cobertura vegetal hacia usos de suelo agrícolas y urbanos, a través de estas áreas de investigación se pueden generar datos y cartografía que contribuya a la toma de decisiones en municipalidades e instituciones responsables del ordenamiento y la administración territorial.

- Interpolación de datos espaciales: Con esta línea de investigación se espera evaluar metodologías de análisis espacial que permitan analizar y modelar la distribución espacial. 
- Unidades de Paisaje: El estudio del paisaje debe ser incluido en todo proyecto de desarrollo, para determinar su calidad frente al ejercicio de ciertas actividades, como para adoptar medidas orientadas a la preservación y protección del espacio natural.

- Ecología de Paisaje: Esta disciplina estudia los paisajes tanto naturales como antrópicos prestando especial atención a los grupos humanos como agentes transformadores de la dinámica físico-ecológica de éstos. Es una de las herramientas en la evaluación del impacto ambiental de las obras y actividades humanas y se usa principalmente en la ordenación del territorio.

- Desarrollo de aplicaciones de programación: A través de esta línea de investigación, se pueden desarrollar aplicaciones que faciliten el uso y análisis de datos espaciales, elaboración de cartografía, análisis espacial entre otras aplicaciones.

En la Tabla 3 se presenta el número de estudiantes de la MOGT2 por cada una de las líneas de investigación seleccionadas.

Tabla 3. Líneas de Investigación de los Estudiantes de la II Promoción MOGT

\begin{tabular}{|c|l|c|}
\hline No. & Área de investigación & $\begin{array}{c}\text { Estudiantes } \\
\text { interesados }\end{array}$ \\
\hline $\mathbf{1}$ & Cartografía: Atlas Temáticos & 1 \\
\hline $\mathbf{2}$ & Cartografía: Recursos Naturales & 1 \\
\hline $\mathbf{3}$ & Cartografía Turística & 1 \\
\hline $\mathbf{4}$ & Cartografía de Riesgos & 1 \\
\hline $\mathbf{5}$ & Localización de equipamientos & 2 \\
\hline $\mathbf{6}$ & Red Geodésica & 2 \\
\hline $\mathbf{7}$ & Dinámica de cobertura del suelo aplicando de técnicas de teledetección & 2 \\
\hline $\mathbf{8}$ & Incendios Forestales & 1 \\
\hline $\mathbf{9}$ & Aplicaciones de Ordenamiento Territorial & 1 \\
\hline $\mathbf{1 0}$ & Definición de las unidades de paisaje & 1 \\
\hline $\mathbf{1 1}$ & Análisis de la dinámica de uso del suelo & 1 \\
\hline $\mathbf{1 2}$ & Interpolación de datos espaciales & 1 \\
\hline $\mathbf{1 3}$ & Desarrollo de aplicaciones de programación & 1 \\
\hline $\mathbf{1 4}$ & Ecología de paisaje & 1 \\
\hline $\mathbf{1 5}$ & Uso de teledetección para la creación de datos & 1 \\
\hline
\end{tabular}

Los resultados de las investigaciones de tesis, se espera sean presentados en el mes de octubre de 2010 , durante el "Congreso sobre Tecnologías de la Información" y donde se dará inicio a la Tercera Promoción de la Maestría en Ordenamiento y Gestión del Territorio MOGT3).

\section{BIBLIOGRAFÍA}

1. Jiménez Galo, Abner Josué. Protocolo de Tesis: "Regionalización de Honduras: Hacia la construcción de regiones plan a través del análisis espacial cuantitativo". Tegucigalpa. 2008.

2. Muñoz Pedreros, A. La evaluación del paisaje: una herramienta de gestión ambiental. Revista Chilena de Historia Natural, 77:139-156, $2004 . \quad$ http://www.scielo.cl/scielo.php?pid=S0716078X2004000100011\&script=sci_arttext. 2004. 
3. Sánchez Ramos, Alexis Alejandro. Protocolo de Tesis: Análisis Multitemporal de la Cobertura de la Tierra en la Cuenca el Valle de Jesús de Otoro, entre los años 2000 - 2006, aplicando técnicas de teledetección. Tegucigalpa: 2008.

4. UNAH, UAH, SGJ-UCP. DIAGNOSTICO para la Creación de la Carrera de Ordenamiento y Gestión del Territorio en el Grado de Maestría. Tegucigalpa: 2005.

5. UNAH, UAH, SGJ-UCP. Proyecto de: Plan de Estudios de la Carrera de Ordenamiento y Gestión del Territorio en el grado de maestría. Tegucigalpa: 2005.

6. UNAH. Informes de las Reuniones de la Comisión Técnica de la MOGT. 2008.

7. UNAH. Reglamento Interno de la Maestría en Ordenamiento y Gestión del Territorio. 2008.

8. UNAH. Reglamento Interno para la Elaboración, Presentación y Defensa de la Tesis de Maestría en Ordenamiento y Gestión del Territorio. 2008. 